\title{
The SHIVA01 trial: what have we learned?
}

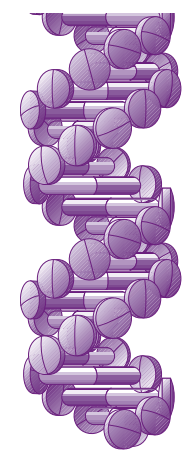

\author{
"Evaluating precision medicine in a prospective trial does not only \\ allow asking efficacy questions of importance for patient care, but \\ also constitute building blocks to enrich knowledge via ancillary \\ studies and curated databases."
}

First draft submitted: 3 April 2017; Accepted for publication: 6 April 2017; Published online: 8 June 2017

Keywords: biomarker $\bullet$ cancer $\bullet$ precision medicine $\bullet$ SHIVA01 • trial design

Our better understanding of the cancer biology has allowed the identification of molecular alterations that drive cancer progression. This knowledge has translated into major therapeutic improvement for the treatment of patients' subgroups whose cancers harbor these molecular alterations. A striking example among others includes the identification of HER 2 amplifications in around $15 \%$ of breast cancer patients [1]. The treatment of HER2positive metastatic breast cancer patients with HER2-targeting agents, such as trastuzumab, has radically modified prognosis with an improved median overall survival from less than 2 years to around 6 years [2]. In fact, most of druggable molecular alterations have been reported to exist across various tumor types, although with differential prevalences [3]. As such, targeting HER2 in HER2-positive metastatic gastric cancer was also demonstrated to improve overall survival [4]. Treating patients with metastatic colorectal cancer or lung cancer harboring an HER2 alteration with HER2-targeting agents was also associated with antitumor activity $[5,6]$. Similar observations were also reported with other altered genes (e.g., $B R A F$ and $R O S 1$ ), giving birth to the concept of precision medicine where patients would be treated according the molecular portrait of their tumor.
The recently published SHIVA01 trial is the first prospective, randomized precision medicine trial comparing targeted therapy based on tumor molecular profile versus treatment by physician's choice in patients with diverse types of metastatic cancer that had failed standard of care treatment [7]. Eleven drugs targeting the PI3K/AKT/mTOR pathway, the RTK/MAPK pathway and the hormone receptor pathway were as such given in patients with various tumor types depending on the result of molecular analyses performed on an on-purpose tumor biopsy of a metastatic site. Given the large variety of tumor types, molecular alterations and drugs, SHIVA01 was not powered to claim whether a specific drug would have any antitumor activity in a specific subgroup of molecularly and histologically characterized patients, as opposed to basket (with an histologic stratification) or umbrella (with a molecular stratification) trials [8]. SHIVA01 belongs to what we can name algorithm-testing trials, since the only parameter that is being evaluated is the efficiency of a prespecified treatment algorithm to improve patients' outcome. The treatment algorithm per se encompasses several features including the technology used to identify druggable molecular alterations, the bioinformatics pipelines and thresholds used

\author{
Christophe Le Tourneau \\ Department of Medical Oncology, \\ Institut Curie, Paris \& Saint-Cloud, France \\ and \\ INSERM U900 Research Unit, Institut \\ Curie, Saint-Cloud, France \\ Tel.: +33144324675 \\ Fax: +33153104026 \\ christophe.letourneau@curie.fr
}

\section{Maud Kamal}

Department of Medical Oncology, Institut Curie, Paris \& Saint-Cloud, France

\section{Ivan Bièche}

Pharmacogenomics Unit, Institut Curie, Paris, France
Future
Medicine
part of 
to call the molecular alterations (e.g., minimum coverage in DNA sequencing and allelic frequency for gene mutations, and the number of copies and amplicon size for gene copy number alterations), the molecular alterations $\leftrightarrow$ drugs matching, as well as prioritization of molecular alterations [9]. The SHIVA01 was negative for its primary end point (i.e., progression-free survival [PFS]) [9]. What have we learned from the results of the SHIVA01 trial?

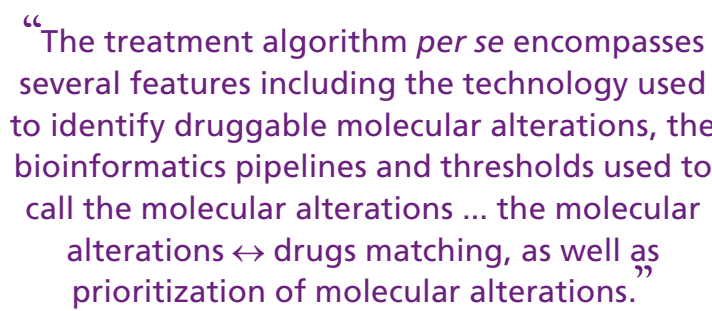

We have learned that it is feasible to run a multicentric fully academic precision medicine trial. Seven hundred and forty one patients were included in 20 months in seven French comprehensive cancer centers. Less than 4 weeks elapsed between the time of the mandatory tumor biopsy and the bioinformatics report [10]. In this aim, genomic and bioinformatics platforms, which used to work for research programs, were engaged in real-time molecular analyses for patients' treatment. The setup of this new diagnostics oriented infrastructure had to be ready and operational, under the supervision of dedicated biologists, before the start of patient inclusions [11]. Two-thirds of patients included in the SHIVA01 trial had a complete molecular profile, including targeted next-generation sequencing for gene mutation analysis, comparative genomic hybridization for gene copy number variation and immunohistochemistry for hormone receptor status evaluation or for the confirmation of any amplified/deleted gene identified via the comparative genomic hybridization [7]. Interestingly, the likelihood to get successful genomic analyses on tumor biopsies was higher if the patient was not receiving cytotoxic chemotherapy at the time of the biopsy; a surgical specimen was taken; and soft tissue metastases were taken in case of computed tomography-guided biopsies [12].

Overall, around $40 \%$ of all included patients had at least one druggable molecular alteration according to the prespecified treatment algorithm [7]. About 26\% of patients were eventually treated. In terms of biology, the most deregulated pathway was not unsurprisingly the PI3K/AKT/mTOR pathway in almost half of patients, followed by the hormone receptor pathway. A few patients had a druggable molecular alteration involving the RTK/MAPK pathway, in which most of clinically validated molecular alterations, such as EGFR, HER2, BRAF, KIT and ALK, belong.
In fact, the overall result of SHIVA01 means the treatment algorithm we had set up to guide therapy was not able to improve outcome of patients with refractory cancer to standard of care as compared with what we do in the clinic in routine. It definitely does not mean the concept of precision medicine, which might not hold true in oncology. Using a different treatment algorithm might have produced different results. Subgroup analyses of SHIVA01 suggested that the concept might be valid for patients whose tumors harbored a molecular alteration involving the RTK/MAPK pathway, with the PFS being almost doubled [7]. This hypothesis needs to be confirmed in subsequent trials.

The results of the prospective randomized SHIVA01 did not confirm the hypotheses generated by nonrandomized clinical studies, including the pilot study by von Hoff et al. [13], the experience from the MD Anderson Cancer Center [14,15] and the MOSCATO trial [16]. All these three studies also belong to algorithm-testing studies in the sense they were not powered to assess the efficacy of a specific drug in a specific subgroup of patients. The efficiency of the precision medicine approach was assessed in all these three studies by comparing the PFS on matched therapy (PFS2) versus the PFS on last prior treatment (PFS1). One major drawback for assessing the primary end point of these studies relies in the way PFS1 has been assessed, since it was not assessed during the study but retrospectively [17]. As an example, PFS1 might have been underestimated if not assessed according to RECIST (standardized Response Evaluation Criteria in Solid Tumors) while PFS2 was. Patients in SHIVA01 were allowed to cross over at disease progression in either arm. The proportion of patients who had a PFS2-to-PFS1 ratio exceeding 1.3 in the subgroup of patients who crossed over in SHIVA01 compared favorably with the results obtained from the von Hoff study and in MOSCATO (37 vs 27 and $33 \%)[13,16,18]$.

More importantly, we have learned from the SHIVA01 trial that treatment algorithms need to be refined. Several critical aspects might explain the negative results of SHIVA01. First, only marketed drugs (and thus first-generation molecularly target agents) used outside their indications were included in the treatment algorithm, given the difficulty to get more attractive drugs in development. At the time of the design of the trial, it was decided that patients with a molecular alteration involving the PI3K/AKT/mTOR pathway would receive everolimus (first-generation mTOR inhibitors), which we now know is not the best drug to target this pathway. Second, drugs were given as single agents, except for patients whose tumors harbored an HER2 amplification or mutation who were treated with trastuzumab and lapatinib. In case of 
multiple druggable molecular alterations, drug combinations might have been more relevant. However, drug combinations are not easy to manage, knowing that almost half of the patients in SHIVA01 treated with single-agent targeted therapy experienced grade 3 or 4 adverse events [7]. Third, the treatment algorithm used in SHIVA01 was unidimensional (i.e., one drug for one molecular target) and did not take into account resistance mechanisms (e.g., KRAS mutations). We now know that drug efficacy might also depend on coexisting molecular alterations. As an example, patients whose tumors harbor a PI3KCA mutation mostly respond to PI3K inhibitors if they do not have a concomitant KRAS mutation. Incorporating information on two molecular alterations to guide to a specific drug is a bidimensional treatment algorithm. It might well be that only the multidimensional treatment algorithm might be able to improve patient's outcome. These treatment algorithms need to accommodate tumor heterogeneity and to incorporate immune parameters as well, given the spectacular efficacy of some immune checkpoint inhibitors in some patients. Only sharing data from different prospective trials might enable us to elaborate such complex treatment algorithms.

Finally, we will continue to learn from SHIVA01 since samples were systematically stored for research purposes. Some ancillary studies using samples or data

\section{References}

1 Slamon DJ, Clark GM, Wong SG et al. Human breast cancer: correlation of relapse and survival with amplification of the HER2-2/neu oncogene. Science 235, 177-182 (1987).

2 Dawood S, Broglio K, Buzdar AU, Hortobagyi GN, Giordano SH. Prognosis of women with metastatic breast cancer by HER2 status and trastuzumab treatment: an institutionalbased review. J. Clin. Oncol. 28(1), 92-98 (2010).

3 Ciriello G, Miller ML, Aksoy BA et al. Emerging landscape of oncogenic signatures across human cancers. Nat. Genet. 45, 1127-1133 (2013).

4 Bang YJ, Van Cutsem E, Feyereislova A et al. Trastuzumab in combination with chemotherapy versus chemotherapy alone for treatment of HER2-positive advanced gastric or gastro-oesophageal junction cancer (ToGA): a Phase III, open-label, randomised controlled trial. Lancet 376(9742), 687-697 (2010).

5 Serra V, Vivancos A, Puente XS et al. Clinical response to a lapatinib-based therapy for a $\mathrm{Li}-$ Fraumeni syndrome patient with a novel HER2V659E mutation. Cancer Discov. 3(11), 1238-1244 (2013).

6 Sartore-Bianchi A, Trusolino L, Martino C et al. Dualtargeted therapy with trastuzumab and lapatinib in treatment-refractory, KRAS codon 12/13 wild-type, HER2-positive metastatic colorectal cancer (HERACLES): from SHIVA01 have been completed, and many others are ongoing $[19,20]$. Evaluating precision medicine in a prospective trial does not only allow asking efficacy questions of importance for patient care, but also constitute building blocks to enrich knowledge via ancillary studies and curated databases. Given the results of the subgroup analyses and of the cross-over data, we are now launching the SHIVA02 trial that will be funded by the MSD Avenir Foundation. In this study, we will focus on the RTK/MAPK pathway, and patients will be taken as their own controls, both PFS1 and PFS2 being assessed during the trial according to RECIST.

Financial \& competing interests disclosure

The SHIVA01 trial was supported by grant ANR-10-EQPX-03 from the Agence Nationale de le Recherche (Investissements d'avenir) and Site de Recherche Intégré contre le Cancer (SiRIC). The molecular platform of the Institut Curie was supported by grants ANR-10EQPX-03 and ANR-10-INBS-09-08 from the Agence Nationale de le Recherche (investissements d'avenir) and the Canceropôle Ile-deFrance. The authors have no other relevant affiliations or financial involvement with any organization or entity with a financial interest in or financial conflict with the subject matter or materials discussed in the manuscript apart from those disclosed.

No writing assistance was utilized in the production of this manuscript.

a proof-of-concept, multicentre, open-label, Phase II trial. Lancet Oncol. 17(6), 738-746 (2016).

7 Le Tourneau C, Delord JP, Gonçalves A et al. Molecularly targeted therapy based on tumour molecular profiling versus conventional therapy for advanced cancer (SHIVA): a multicentre, open-label, proof-of-concept, randomised, controlled Phase II trial. Lancet Oncol. 16(13), 1324-1334 (2015).

8 Le Tourneau C, Kamal M, Alt M et al. The spectrum of clinical trials aiming at personalizing medicine. Chin. Clin. Oncol. 3, 13 (2014).

9 Le Tourneau C, Kamal M, Tsimberidou AM et al. Treatment algorithms based on tumor molecular profiling: the essence of precision medicine trials. J. Natl Cancer Inst. 108(4), pii: djv362 (2015).

10 Le Tourneau C, Paoletti X, Servant N et al. Randomised proof-of concept Phase II trial comparing targeted therapy based on tumour molecular profiling vs conventional therapy in patients with refractory cancer: results of the feasibility part of the SHIVA trial. Br. J. Cancer 111, 17-24 (2014).

11 Servant N, Roméjon J, Gestraud P et al. Bioinformatics for precision medicine in oncology: principles and application to the SHIVA clinical trial. Front. Genet. 5, 152 (2014).

12 Desportes E, Wagner M, Kamal M et al. Prognostic factors of successful on-purpose tumor biopsies in metastatic cancer patients included in the SHIVA prospective clinical trial. Oncotarget 8(1), 1760-1773 (2017). 
13 von Hoff DD, Stephenson JJ, Rosen P et al. Pilot study using molecular profiling of patients' tumors to find potential targets and select treatments for their refractory cancers. J. Clin. Oncol. 28(33), 4877-4883 (2010).

14 Tsimberidou AM, Iskander NG, Hong DS et al. Personalized medicine in a Phase I clinical trials program: the M. D. Anderson Cancer Center Initiative. Clin. Cancer Res. 18, 6373-6383 (2012).

15 Tsimberidou AM, Wen S, Hong DS et al. Personalized medicine for patients with advanced cancer in the Phase I program at MD Anderson: validation and landmark analyses. Clin. Cancer Res. 20, 4827-4836 (2014).

16 Massard C, Michiels S, Ferte C et al. High-throughput genomics and clinical outcome in hard-to-treat advanced cancers: results of the MOSCATO 01 trial. Cancer Discov. doi:10.1158/2159-8290.CD-16-1396 (2017) (Epub ahead of print).
17 Doroshow JH. Selecting systemic cancer therapy one patient at a time: is there a role for molecular profiling of individual patients with advanced solid tumors? J. Clin. Oncol. 28, 4869-4871 (2010).

18 Belin L, Kamal M, Mauborgne C et al. Randomized Phase II trial comparing molecularly targeted therapy based on tumor molecular profiling versus conventional therapy in patients with refractory cancer: cross-over analysis from the SHIVA trial. Ann. Oncol. 28(3), 590-596 (2016).

19 Lebofsky R, Decraene C, Bernard V et al. Circulating tumor DNA as a non-invasive substitute to metastasis biopsy for tumor genotyping and personalized medicine in a prospective trial across all tumor types. Mol. Oncol. 9(4), 783-790 (2015).

20 Lefebvre C, Bachelot T, Filleron T et al. Mutational profile of metastatic breast cancers: a retrospective analysis. PLoS Med. 13(12), e1002201 (2016). 